\title{
HIPÓTESIS SOBRE LA CONVERGENCIA ECONÓMICA: UNA REVISIÓN DE LOS ENFOQUES UTILIZADOS
}

ISSN 2219-6722

ISSNE 2222-2707

\author{
CRISTIAN RABANAL, Departamento de Matemática y Estadística. \\ Universidad Nacional de Río Cuarto (UNRC). \\ Consejo Nacional de Investigaciones Científicas y Técnicas (CONICET). Argentina
}

\section{RESUMEN}

El propósito de este artículo es realizar una diferenciación de las hipótesis alternativas que habitualmente se realizan sobre la convergencia, a partir de una revisión de los principales procedimientos metodológicos propuestos. Asimismo, se revisa la evidencia teórica y empírica que soporta a cada una de ellas.

Si bien actualmente todas las metodologías continúan siendo ampliamente utilizadas, se observa un marcado interés por parte de los investigadores en el estudio de las técnicas espaciales que permitan incluir fenómenos derivados de la dependencia espacial, tales como efectos umbrales y externalidades.

Palabras clave: convergencia, regresiones a la Barro, metodología de Quah, Convergencia estocástica, dependencia espacial. 


\title{
ASSUMPTIONS ABOUT ECONOMIC CONVERGENCE: A REVIEW OF APPROACHES USED
}

ISSN 2219-6722

ISSNE 2222-2707

CRISTIAN RABANAL, Departamento de Matemática y Estadística. Universidad Nacional de Río Cuarto (UNRC).

Consejo Nacional de Investigaciones Científicas y Técnicas (CONICET). Argentina

\begin{abstract}
ABSTRAC
The goal of this paper is to distinguish among alternatives convergence hypothesis from the foremost procedures proposed. Besides, empirical evidence is reviewed.

Although each procedure is used nowadays, researchers seem to be keen on spatial techniques to include those phenomenons arising from spatial dependence such as threshold and externalities.
\end{abstract}

Key Words: convergence, Barro's regressions, Quah's methodology, stochastic convergence, spatial dependence. 


\section{INTRODUCCIÓN}

El estudio del crecimiento económico fue uno de los temas dominantes en el desarrollo teórico de la economía, desde mediados de los cincuenta y hasta principios de los noventa. Durante dicho período, se desarrollaron dos posturas teóricas claramente diferenciadas: los modelos de crecimiento exógeno, cuyos representantes precursores fueron Solow (1956), Swan (1956) y los modelos de crecimiento endógeno (Romer, 1986). Dichas modelizaciones arribaban a conclusiones completamente diferentes. Mientras los primeros vaticinaban por la convergencia de las economías, los segundos ofrecían argumentos que apuntaban a la divergencia. La razón fundamental para tales conclusiones emergía del supuesto central en relación a la presencia -modelos exógenos- o ausencia -modelos endógenos- de rendimientos decrecientes en los factores acumulables.

En dicho contexto, durante la década del noventa, el debate teórico abandonó la cuestión del crecimiento, estudiada de manera exclusiva, y comenzaron a surgir numerosos estudios en relación a la convergencia económica. La importancia del tema derivó rápidamente en un asunto epistemológico, ya que, al ser considerado el estudio de la convergencia como una prueba empírica, se podía pensar en ella como un contraste que juzgue sobre la verdad de los modelos teóricos de crecimiento desarrollados hasta entonces, los cuales resultaban claramente antagónicos.

No obstante, ni bien comenzada la etapa de contrastación, donde el estudio de la convergencia estuvo en auge, comenzaron a proponerse diferentes maneras de estudiarla, en función de la gran cantidad de métodos estadísticos disponibles.

Siguiendo a Rabanal (2012), Islam (2003) reconoce cuatro formas diferentes en las que se aborda el tema de la convergencia. La primera de ellas, fue la propuesta basada en datos de sección cruzada, popularizada por Sala-iMartin (1992) y Mankiw et. al. (1992). Dicho análisis permitió, conocido como regresiones a la Barro, no sólo permitió estudiar la relación de convergencia en términos de crecimiento y dotación inicial de renta, sino también incluir variables vinculadas a ratios de inversión y capital humano fundamentalmente. Las principales críticas a este enfoque provienen de su propia formulación. En particular, autores como Quah (1995) han sugerido que dicho contraste es en realidad un contraste de raíz unitaria, que se encuentra dominado por el fenómeno de reversión a la media (Falacia de Galton). Por otra parte, Arbia y Deglistudi (2004), ha criticado el enfoque por el carácter estático del mismo, lo que no permite el estudio de la dinámica del proceso de convergencia.

Una segunda metodología implementada en el estudio de la convergencia se ha desarrollado a partir de modelos de datos de panel (Islam, 1995). Este procedimiento permitió la incorporación de algunas diferencias regionales y controlar las características inobservables que las conforman (Aixalá y Simón, 2003). Una importante contribución desde este enfoque han sido los estudios sobre la convergencia de la productividad total de los factores -PTF- (Dollar y Wolff, 1994). Si bien este enfoque es descripto aisladamente en esta introducción, los datos de panel son en realidad abordajes híbridos de datos temporales y de sección cruzada.

El tercer desarrollo para analizar la temática ha sido el de Quah (1993, 1995,1996), cuya técnica puede considerarse aisladamente, pues no comparte elementos en común con las restantes. La principal motivación del autor es el estudio de la dinámica de la convergencia. La herramienta adoptada es la inspección gráfica de los núcleos estocásticos, lo que permite verificar la evolución de la distribución del PBI per cápita de los diferentes países en cada año. No obstante, la mencionada técnica presenta una importante debilidad: la selección del ancho de banda óptimo. Un ancho de banda inadecuado, puede dar lugar a una distribución artificialmente unimodal. Aunque las cadenas de transición de Markov permiten estudiar la convergencia en términos analíticos, también plantean el desafío de la elección del número de estados a considerar, lo que resulta subjetivo. 
Finalmente, el análisis de series de tiempo ha permitido estudiar la convergencia, entendida como un proceso estacionario. Esto es, el PBI per cápita de los países es expresado en relación a una economía, lo que permite homogeneizar las diferencias. Luego, los estudios se concentran es examinar si dichas diferencias siguen procesos estacionarios en el largo plazo mediante diversas pruebas de raíz unitaria (Lee, 1997). También ha sido frecuente el uso de técnicas de cointegración para el contraste (Bernard y Durlauf, 1995) y el análisis de convergencia en la tendencia y los ciclos (Carvalho y Harvey, 2003).

\section{LA CONVERGENCIA ABSOLUTA VS. CONVERGENCIA CONDICIONAL}

Aunque la convergencia económica puede ser interpretada operativamente de diversas maneras, en función de la aproximación utilizada para su estudio, a nivel teórico se resume básicamente en una proposición según la cual existe una relación inversa entrcve la tasa de crecimiento de la renta per cápita y el nivel inicial de la misma, para un conjunto de regiones o países dado y un período de tiempo determinado. Cuando este proceso se verifica independientemente de las condiciones iniciales se lo denomina convergencia absoluta (o convergencia incondicional). En consecuencia, esta hipótesis puede implicar a un grupo heterogéneo de países o regiones, desde el punto de vista de sus características estructurales. Este augurio resultó alentador para los países más pobres, puesto que suponía que podrían converger al nivel de ingreso de los países más ricos.

No obstante, el equilibrio de largo plazo de cualquier economía se encuentra condicionado por sus propias características estructurales como la tecnología disponible, el crecimiento poblacional y las políticas públicas implementadas entre otras, por lo que la convergencia absoluta requiere también la convergencia de esas características estructurales de un conjunto de países determinado (Galor, 1996). En este sentido, algunos autores atribuyen a las reformas estructurales un punto de partida inevitable para que los países de menor ingreso relativo puedan iniciar un proceso que les permita reducir la brecha respecto a los más ricos. En este sentido, Sachs y Warner (1995), presentan evidencia de una condición suficiente para la convergencia absoluta, que radica en seguir políticas económicas "razonablemente eficientes" en lo referido a la apertura comercial y a la protección de los derechos de propiedad privada. A los países que cumplen con dicha condición los denominan "países cualificados". En consecuencia, un país puede ser clasificado como no cualificado por no tener un grado de apertura comercial "adecuado" ( que a criterio de los autores esto se da cuando la mayor proporción de importaciones proceda de cuotas de importación, cuando la estructura económica es socialista o existe una prima del $20 \%$ o más en el mercado cambiario no oficial, para el caso de la década del setenta o del ochenta), o bien por no respetar los derechos de propiedad (por ejemplo, tener una estructura económica socialista, presentar disturbios domésticos presentados por revoluciones, golpes de estado, o conflictos armados-, privación de derechos civiles y políticos). Sachs y Warner (1995) detectan en su estudio, que el grupo de países cualificados presenta una fuerte tendencia hacia la convergencia absoluta. No obstante, China constituye la única excepción en el análisis de los autores, que a pesar de no haber sido clasificado con tal, presenta convergencia incondicional. De cualquier modo, enfatizan las fuertes reformas emprendidas por ese país asiático a finales de la década del setenta.

Desde el punto de vista de la convergencia absoluta, hay también una consideración positiva del rol de la globalización y los desarrollos tecnológicos junto con la apertura comercial de las economías, ya que se considera que han facilitado la interacción económica de países distantes entre sí. Dollar (2001) y Lindert y Williamson (2003) han proporcionado evidencia de convergencia entre los niveles de ingresos de países en desarrollo que se han globalizado y el nivel de ingresos de países desarrollados. En este sentido, la globalización no sólo ha disminuido las distancias, sino que también ha acortado los tiempos para que 
nuevas tecnologías puedan llegar a países rezagados en materia económica, lo que constituye por sí mismo un aliciente a la convergencia o a la disminución de las desigualdades. También Villaverde y Maza (2011) sostienen que la globalización ha sido uno de los principales conductores del crecimiento económico, fomentando la convergencia en el ingreso per cápita. El estudio de estos autores incluye 101 países con diferentes grados de desarrollo, y abarca el período 1970-2005.

En un esfuerzo por medir cuestiones derivadas de diferentes facetas de la globalización, Álvarez Ayuso et. al. (2009) estudian cómo la integración económica, política y social ha influido en el proceso de convergencia para los países latinoamericanos durante el período 1970-2005. La integración económica abarca aspectos como el volumen de comercio exterior, la inversión extranjera directa y la existencia de barreras al comercio o a la movilidad de capitales. Por su parte, la integración política considera aspectos como la pertenencia a organismos internacionales, contribuciones a las misiones de paz de la ONU, el número de embajadas y la ratificación de tratados internacionales. Finalmente, la integración social incluye datos de viajes turísticos, tráfico telefónico internacional, envíos de dinero y números de usuarios de internet. Con esa información, los autores construyen un índice económico, otro político y uno social analizando los respectivos impactos sobre la convergencia. Los resultados muestran que, para todo el período de estudio, la faceta económica y social ha afectado de manera positiva y significativa a la reducción de la brecha de ingresos entre países pobres y ricos. Este resultado es consistente con el trabajo que Dreher (2006) desarrolló examinando el impacto de la globalización sobre el crecimiento económico, para un contexto mundial. No obstante, la integración política no ha arrojado un resultado concluyente. La evidencia empírica desarrollada en la década de los ochenta, no convalida en general la hipótesis de convergencia absoluta. Si bien el trabajo de Rostow (1980) concluye a favor de la convergencia, luego de utilizar un análisis combinado de series de tiempo y datos de sección cruzada, los trabajos posteriores comenzaron a enfatizar sobre cuestiones vinculadas al agrupamiento en las muestras y diferencias estructurales.

Abramovitz (1986) publicó un artículo en el que postulaba que la diferencia entre los niveles de productividad de los países genera un fuerte potencial para una posterior convergencia. Esto sucede, según el autor, siempre que los países cuenten con la capacidad social de absorber las tecnologías más avanzadas. De esta forma se enfatiza la preponderancia de la tecnología y las implicancias de los modelos de crecimiento exógeno en el proceso de convergencia. Se asume también que los países de menor desarrollo tecnológico pueden imitar a un bajo costo los desarrollos tecnológicos más avanzados. Es precisamente, en este último punto, donde yace una de las condiciones más significativas para la convergencia al menos en tres aspectos. En primer lugar, por la reducción de los plazos temporales con los que se difunden los nuevos desarrollos tecnológicos, lo que permite a las regiones más pobres disponer de los más recientes desarrollos en períodos más breves. En segundo lugar, por la disminución de costos de imitación emergente de las economías de escalas, originadas en los mercados de mayor tamaño que surgen por la propia dinámica de integración que la globalización genera en la conformación de bloques regionales. Adicionalmente, la aparición de dichos bloques ha permitido avanzar hacia acuerdos arancelarios inter-bloques, lo que ha facilitado la eliminación recíproca de gravámenes que pesan sobre las importaciones. En tercer lugar, y probablemente el aspecto más importante de manera directa, la adquisición de nuevas formas de producir conlleva implícito necesariamente una mejora en la productividad laboral. Esto último, implica poner un límite a la desigualdad atribuible a las diferencias que se originan en la forma de producción, en especial manufacturas de origen industrial.

En la misma dirección, seis meses más tarde, Baumol (1986) examinó la convergencia entre 16 países industrializados para el período 1870-1979. Entre sus principales resultados, el autor muestra una elevada convergencia entre las naciones industrializadas. Esta relación también se convalidó para economías con 
planificación central. No obstante, al ampliar la muestra encontró una gran dispersión en el conjunto general de datos. Esto lo llevó a acuñar el término club de convergencia, según la cual los países convergen con otros que comparten ciertas características estructurales. Estos hallazgos fueron cuestionados por DeLong (1988), quien los consideró espurios por el sesgo que presentaba la selección de la muestra. Según éste autor se trata de una selección ex post de países que al momento de ser analizados por Baumol (1986) ya eran ricos y contaban con un nivel de desarrollo satisfactorio.

Otro tema de importancia en relación a los estudios de convergencia fue el impacto que significó la Segunda Guerra Mundial. El mismo se tornó particularmente importante, ya que la destrucción de infraestructura y stock de capital en algunos países afectados creó las condiciones según las cuales, la teoría supone que los países afectados deberían crecer más a prisa que el resto de países que no sufrieron daños -siempre que previo al shock compartieran un conjunto de características (fundamentals) similares. - para lograr reconstituirse y alcanzar la convergencia. En este sentido, Li y Papell (1999), encuentran convergencia utilizando un enfoque de series de tiempo para 16 países de la OCDE y atribuyen a la Segunda Guerra Mundial la mayor causa de los desplazamientos estructurales en el producto relativo. También Cook (2002) ha estudiado y medido el efecto de la Segunda Guerra Mundial sobre el proceso de convergencia con una muestra de 42 países y para el periodo 1950-1990. Particularmente, examinó la velocidad a la que los países industrializados se restituyeron de los daños de la guerra, a partir de la reducción del stock de capital sufrida en algunos países, como Japón y otros estados de Europa ${ }^{5}$. El autor encuentra evidencia favorable a la hipótesis de convergencia, a lo que presenta como un resultado completamente previsto en el modelo de Solow y Swan (1956). Cook (2002), da cuenta de una velocidad de convergencia entre el 4\% y el 6\% para el período de estudio, siendo esos valores significativamente más elevados al tradicional $2 \%$. Esto implica que los países que sufrieron daños por la guerra, materializados principalmente a través de la disminución del stock de capital disponible, han tenido un crecimiento más rápido en la productividad para alcanzar el sendero de largo plazo.

Recientemente, Rodrick (2011a, 2011b) ha encontrado evidencia a favor de un proceso de convergencia incondicional a partir de 1990 en la productividad del trabajo del sector de industrias manufactureras y para un amplio grupo de países desarrollados y en desarrollo. No obstante, al igual que Abramovitz (1986), el autor advierte que la tasa a la cual las economías en desarrollo convergerán estará "determinada por las habilidades para absorber ideas y conocimientos desde la frontera tecnológica” (Rodrick, 2011a; p. 3).

Una de las ideas más resistidas para la hipótesis de convergencia incondicional es la condición de equifinalidad. Esto es, tres economías con recorrido completamente diferente en el ínterin, pueden acabar convergiendo con patrones completamente distintos. La posibilidad de que un gran número de situaciones pueda conducir a un mismo resultado en términos de $\beta$-convergencia se ha denominado por algunos autores como equifinalidad (Arbia, 2004).

La idea de convergencia cuenta con el apoyo del premio Nobel en economía, Michel Spence, quien sostiene que el mundo se encamina "por primera vez en 250 años hacia un patrón de convergencia en lugar de divergencia” (Spence, 2011; p. 4). Rabanal (2012), también ha encontrado evidencia de convergencia incondicional en sub muestras pequeñas y homogéneas de países para el período 1950-2009, a partir de la aplicación de la ecuación tradicional de $\beta$-convergencia. Esto se vuelve más evidente, cuando se van considerando valores incrementales para la dotación inicial de renta. Adicionalmente, el autor presenta la metodología de Quah. Haciendo uso del ancho de banda óptimo propuesto por Silverman (1986) para analizar la transición dinámica de las funciones de densidad estimadas. Desde la estimación de cadenas de Markov de un paso de 4 y 5 estados, la evidencia sugiere que un proceso de convergencia tuvo lugar desde 1950 hasta los primeros años de la década del setenta.

5 Alemania, Austria, Bélgica, Dinamarca, España, Finlandia, Francia, Grecia, Italia, Luxemburgo, Noruega, Países Bajos y Reino Unido. 
Por otra parte, la hipótesis de convergencia condicional hace referencia a la relación inversa entre la tasa de crecimiento de la renta per cápita y el nivel inicial de la misma, para un conjunto de regiones o países dado con características estructurales similares y un período de tiempo determinado.

Barro (1991) ha encontrado evidencia a favor de la convergencia condicional. No obstante, la tasa de convergencia no es rápida, siendo que la brecha entre los países pobres y ricos disminuye cerca del 2\% por año (Barro y Sala-i-Martin, 1992). Mankiw et. al. (1992), extendieron el modelo para considerar el capital humano, lo que implicaba ciertos procesos de convergencia de larga duración ó amplios períodos de catching-up.

La consideración de variables adicionales no incluidas en el análisis incondicional, que forman parte de los fundamentos de cada economía, hacen que sea probable encontrar este tipo de convergencia entre regiones de un mismo país, en virtud que comparten un mismo marco institucional y se encuentran expuestas a un mismo riesgo sistemático (Sala-i-Martin, 1996). A nivel países, algunos autores, valiéndose de los modelos de crecimiento endógeno vaticinan la divergencia (Rebelo, 1991). La razón fundamental es que existen factores acumulables que no presentan rendimientos decrecientes. Desde este punto de vista no existen motivos para esperar procesos de convergencia. En este sentido, Romer (1986) y Lucas (1988), por ejemplo, consideran en sus análisis la presencia de externalidades y los rendimientos de las inversiones como una función creciente del stock de capital en un momento inicial.

\section{LOS CLUBES DE CONVERGENCIA}

El estudio de Baumol (1986) fue el primero en utilizar el término club de convergencia, para hacer referencia a países que convergen con otros que comparten ciertas características estructurales. En su artículo, Baumol agrupa a los países en tres, encontrando convergencia sólo en el grupo de los más ricos quienes pasan a conformar un club de convergencia.

No debe confundirse la convergencia condicional con la noción de club de convergencia. De acuerdo con Islam (2003; p. 315), "la idea de club de convergencia está basada sobre modelos que admiten múltiples equilibrios". Por el contrario, en la convergencia condicional, el equilibrio es único para cada unidad de observación. En consecuencia, en un club de convergencia, algunas economías no convergerán al mismo estado estacionario, pero un grupo si lo harán a un estado estacionario particular. Por esta razón, los países de cada club convergerán entre sí, pero no habrá convergencia entre diferentes clubes.

Chatterji y Dewhurst (1996) han demostrado que la ecuación tradicional de beta convergencia, puede ser modificada para considerar la posible existencia de clubes de convergencia. En la versión modificada, la variable clave es el logaritmo natural del ingreso per cápita relativo, donde existen básicamente dos posibilidades. La primera de ellas es considerar un país líder y calcular el ingreso de todos los demás países en función del líder. La limitación de esta alternativa resulta evidente si el país líder pierde tal condición en algún momento del período de tiempo analizado. Por esta razón, resulta conveniente considerar el ingreso per cápita relativo a la media del conjunto de países que conforman el grupo de interés. Bajo estas consideraciones, la reformulación de Chatterji y Dewhurst (1996) permite reformular a clásica ecuación de beta convergencia de la siguiente forma:

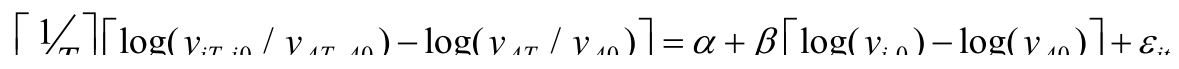

donde $A$ hace referencia a la media, T al período de tiempo estudiado, y 0 el momento inicial de estudio. Si se denota como $z_{i T}=\left[\log \left(y_{i t} / y_{i \theta}\right)-\log \left(y_{A T}-y_{A \theta}\right)\right]$ y $z_{i 0}=\left[\log \left(y_{i o}\right)-\log \left(y_{A \theta}\right)\right]$, la expresión (1) se puede reducir a: 


$$
\lceil 1 / T\rceil z_{i t}=\ell
$$

Para permitir la existencia de múltiples clubes de convergencia $(x)$, se puede reescribir (2) con la reformulación siguiente:

$$
[1 / T] z_{i t}=\sum_{x=1}^{X} \beta_{x}\left(z_{i, t-T}\right)^{k}=\phi(z
$$

De acuerdo con Villaverde (2004), la forma funcional de $\Phi$ dependerá fundamentalmente de los datos.

La figura No. 1 muestra el gráfico de $z_{i t} \operatorname{contra} z_{i 0}$, donde se puede comparar la brecha de cada economía respecto a la media en el año final e inicial. En la misma puede distinguirse claramente diferentes situaciones. Si la situación inicial se encuentra entre los puntos $E_{1}$ y $E_{3}$, la brecha hacia el promedio tenderá a achicarse y la convergencia tendrá lugar en el punto $E_{0}$. Una brecha mayor a la representada en $E_{1}$ o $E_{3}$ (es decir puntos a la derecha de $E_{1}$ o a la izquierda de $\mathrm{E}_{3}$ ) tenderá a incrementarse con el transcurso del tiempo, y la convergencia se producirá en $E_{2} \mathrm{o} E_{4}$.

Figura 1: Club de convergencia. Niveles de ingreso per cápita relativo a la media del grupo de pertenencia

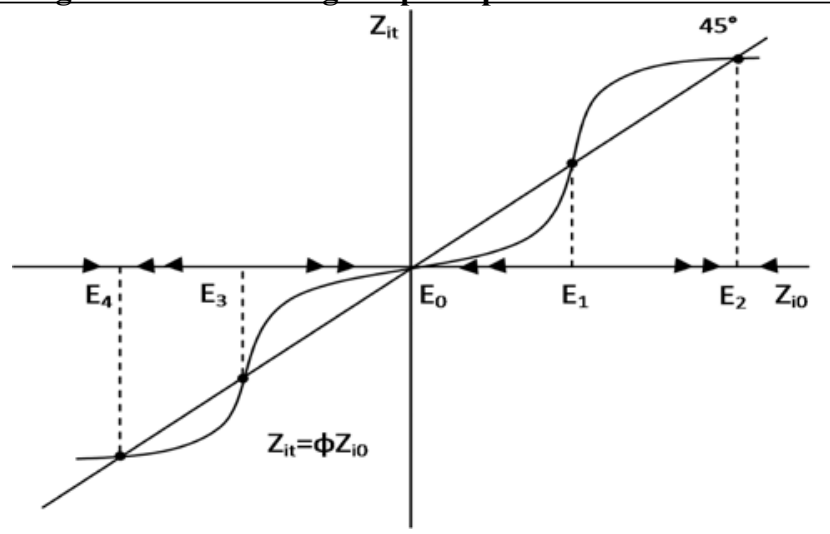

Fuente: Elaboración propia sobre la base de Villaverde (2004).

Mientras que a nivel teórico la diferencia entre convergencia condicional y club de convergencia resulta evidente, la diferenciación empírica no es sencilla. Islam (2003) reconoce que esta dificultad se debe a los problemas para determinar el criterio a utilizar en la agrupación de países para el testeo de un club de convergencia. De cualquier modo, los países que integran un club son sólo aquellos miembros de un grupo que muestran convergencia, y no aquellos países que divergen para una categoría de ingresos dada (Qing, 1999). 
Kristensen (1982) encontró indicios de convergencia entre los países más ricos y divergencia entre los más pobres. Concretamente, el autor trabajando con datos de sección cruzada agrupó a los países entre siete grupos posibles según sus niveles de ingreso en 1974. La relación entre las tasas de crecimiento en el período 1974-1978 y sus niveles de ingreso para los diferentes grupos puede representarse adecuadamente como una parábola convexa, lo que daba cuenta de la divergencia para los tres grupos iniciales de menores ingresos y convergencia para los restantes grupos de mayores ingresos. Estos resultados de divergencia entre los más pobres y convergencia entre los más ricos fueron avalados por estudios posteriores como el de Chenery y Syrquin (1986) y el de Qing (1999).

Luego de la acuñación del término por parte de Baumol, la idea de clubs de convergencia fue retomada luego por numerosos autores, entre ellos Ben David (1997), quien encontró evidencia de clubes de convergencia entre los países más ricos, aunque señala que, si se tomara una muestra aleatoria desde los mismos, la probabilidad de encontrar convergencia sería menor a la probabilidad de encontrar divergencia. Sin embargo, este autor ha advertido sobre la sensibilidad de los resultados a la composición de la muestra, lo que constituye uno de sus aportes fundamentales. En particular, motiva al autor conocer el cuál es subgrupo de países pobres y el subgrupo de países ricos, haciendo referencia a que con el paso del tiempo se han considerado diferentes agrupamientos. Mientras que por ejemplo Kristensen (1982) consideró siete subgrupos, Baumol (1986) tomó en consideración sólo tres. Dependiendo de la forma en que se agrupen los países, el autor también sugiere la existencia de convergencia entre los países más pobres.

\section{MEDICIÓN DE LA CONVERGENCIA}

\subsection{Convergencia beta, sigma y gamma}

La noción de convergencia beta refiere a la idea de que los países que detentan una menor renta en un período inicial $t_{0}$ tendrán un crecimiento más elevado durante el período $t_{0}-t_{l}$ que aquellos países que partían con dotaciones más alta de renta en el comienzo del proceso. Este concepto fue ampliamente difundido en los trabajos de Barro y Sala-i-Martín (1991, 1992). El contraste habitualmente utilizado ha sido la estimación de la siguiente ecuación:

$$
\left./_{T}\right]\left[\log \left(y_{i, T}\right)-\log \left(y_{i, 0}\right)\right]=\alpha+\beta \log \left(y_{i, 0}\right)+
$$

donde $\alpha$ representa un parámetro tecnológico y $\beta$ la tasa de convergencia, que se asume menor a la unidad para evitar adelantamientos sistemáticos y $\varepsilon$ residuo. $T$ es la longitud del período, $y_{i, 0}$ la renta de la región $i$ en el instante inicial e $y_{i}, T$ la renta de la región $i$ en el instante final. Una situación de adelantamiento sistemático tendría lugar si los países de menor renta relativa en un período inicial $\mathrm{t}_{0} \operatorname{lograran}$ crecer, en un determinado período $t_{0}-t_{l}$, sobrepasando a los países de mayor renta relativa en $t_{0}$ y a continuación, en el período siguiente, $t_{1}-t_{2}$, los países de menor renta relativa en $t_{1}$ crecerían hasta superar a los países de mayor renta relativa en $t_{l}$. De esta forma, el proceso podría continuar indefinidamente en el tiempo.

Para el caso de convergencia condicional de tipo beta es habitual incorporar en (4) variables que refieran a las condiciones estructurales de largo plazo de la economía, tales como educación, niveles de ahorro, variables demográficas e incluso institucionales.

La cuestión central en esta metodología, radica en determinar la magnitud y significatividad del coeficiente $\beta$. Si el mismo resultara negativo e individualmente significativo se podría admitir un proceso de convergencia incondicional en una formulación similar a la expresión (4) o condicional para el caso que se incorporen otras variables. En cualquier caso, también sería posible calcular la velocidad del proceso de 
convergencia de la siguiente manera:

$$
V=-\operatorname{Ln}(1+T \beta
$$

Por otra parte, resulta interesante analizar también la $\sigma$-convergencia. Este concepto se refiere a la disminución de la dispersión del PBI per cápita a lo largo del tiempo. La $\beta$-convergencia es una condición necesaria pero no suficiente para que tenga lugar un proceso de $\sigma$-convergencia. La razón es que muchos disturbios, tales como "guerras y shock agrícolas o del petróleo afectan de manera diferenciada a las economías” (Barro y Sala-i-Martin, 1991: p.113). Dichas perturbaciones provocarán un incremento en la varianza de $\varepsilon_{i}$

Analíticamente, la $\sigma$-convergencia ha sido representada habitualmente de dos formas alternativas. La primera de ellas, hace referencia a la desviación típica de los logaritmos:

$$
D T\left(\ln _{t}\right)=\left(\frac { 1 } { n } \sum _ { i = 1 } ^ { n } \left(\ln \mathrm{y}_{i t}-\overline{\ln \mathrm{y}_{i}}\right.\right.
$$

mientras que la segunda posibilidad radica en el cálculo del coeficiente de variación:

$$
C V_{t}=\frac{\left(\frac{1}{n} \sum_{i=1}^{n}\left(\ln \mathrm{y}_{i t}-\overline{\ln \mathrm{y}_{t}}\right)\right.}{\overline{y_{s}}}
$$

Ahora bien, a pesar de tratarse de medidas de dispersión muy similares, algunos autores como Dalgaart y Vastrup (2001) han demostrado que pueden llevar a conclusiones contradictorias. Utilizando datos de 121 países desde la Penn World Table, los autores encuentran dicho inconveniente y lo asignan a que tales medidas establecen diferentes pesos para la performance de crecimiento de cada país. Por este motivo, en los estudios abocados a la convergencia como reducción de las desigualdades, suelen utilizarse algunas mediciones complementarias como el coeficiente de Gini, el índice de Theil o la curva de Lorenz. Asimismo, otros autores han centrado la atención en la polarización en lugar de la desigualdad. En este sentido, Esteban y Ray (1994) afirman que las medidas de desigualdad han fracasado en distinguir de forma correcta la convergencia hacia la media mundial y la clusterización hacia medias locales. Los autores enfatizan que las medidas de polarización son naturalmente globales, mientras que las de desigualdad no. Por ejemplo, el Principio de Transferencia de Dalton, que subyace el análisis de la Curva de Lorenz, establece que, partiendo de cualquier distribución del ingreso, cualquier transferencia de ingresos de un individuo hacia otro más rico que él, debe incrementar la desigualdad. Este principio es una clara representación de un criterio local.

Por otra parte, Sala-i-Martin (1996) muestra que la relación entre la $\sigma$-convergencia y la $\beta$-convergencia puede representarse adecuadamente por la siguiente expresión:

$$
\sigma_{t}^{2} \cong(1-\beta)^{2} \sigma_{t-1}^{2}+\sigma_{\mu}^{2}
$$

de donde resulta que la $\beta$-convergencia es una condición necesaria para que la varianza no se incremente con el paso del tiempo.

Finalmente, el concepto de gamma convergencia, ha sido propuesto por Boyle y McCarthy (1997 y 1999) con el objetivo de comprobar la beta convergencia. Para ello, los autores desarrollaron este nuevo concepto de una forma más general, proponiendo el estudio de los cambios que se produzcan en un ranking u 
ordenación de todas las unidades, según un índice de concordancia de rangos de Kendall en su versión binaria. De esta forma, el indicador puede ser obtenido de la siguiente forma:

$$
C R_{t}=\frac{\operatorname{var}\left[R(y)_{i t}-R(y)_{i 0}\right]}{\operatorname{var}\left[2 R(y)_{i 0}\right]}
$$

$R\left(y_{i}\right)$ es el ordenamiento actual de la renta per cápita para el período $t$. Cuando el período de estudio no registre cambios, el valor máximo a alcanzar estará dado por el denominador. De esta forma, el índice asume valores entre cero y uno, donde un valor de uno para todo el período indicaría ausencia de convergencia durante el mismo. La metodología, considera que el año de partida, el índice asume el valor de uno. La distribución del indicador es una chi-cuadrado con $n-1$ grados de libertad. Además de la versión binaria, también es posible computar al índice de forma multi anual.

\subsection{La metodología de Quah}

Laa propuesta de Quah $(1993,1995,1996)$ no sólo plantea críticas a la metodología sugerida por Sala-iMartín (1991, 1992, 1996), sustentada en modelos de regresión a la Barro, sino que también significó el aporte de una nueva perspectiva para el estudio de la convergencia.

Los principales resultados de Quah (1995) prevén la polarización del ingreso de los países, y la conformación de sólo dos clases, países ricos y países pobres, cuya distancia entre los mismos será cada vez mayor.

En relación a las críticas, alerta por la existencia de deficiencias provenientes de las regresiones a la Barro, y la tasa de crecimiento que se desprende de las mismas. En particular, Quah asegura que existe una tendencia central que agrupa las estimaciones de las betas estimados en torno al 2\%. Según Quah (1995), esta “tasa mágica" es invariante en términos de período de tiempo y conjunto de países estudiado. En consecuencia, surge la conjetura sobre cuál es el papel que dicha tasa desempeña en la dinámica de los procesos de convergencia y si se trata de una estructura subyacente de la economía o no. Quah (1995) muestra que en realidad se trataría de un proceso de raíz unitaria. Para comenzar, parte de la ecuación de beta convergencia tradicional:

$$
T^{-1}\left(Y_{i}(T)-Y_{i}(0)\right)=\alpha-\left(\frac{1-e^{-\beta T}}{T}\right) Y_{i}(0)+\varepsilon_{i}(T)
$$

donde Y representa el logaritmo del PBI per cápita en el país $i, 0$ el instante inicial, $T$ el instante final, $\alpha$ un parámetro tecnológico, $\beta$ la tasa de convergencia. Reescribiendo (10) y haciendo $b T=e-\beta T$ se tiene que:

$$
T^{-1}\left(Y_{i}(T)-Y_{i}(0)\right)=a+b_{T} T^{-1} Y_{i}(0)+\varepsilon_{i}(T)
$$

Multiplicando (11) por $T$, resulta que cuando $\beta$ es 0,02 y $T$ es igual a $10, b T$ es igual a 0,82 . Pero, si $\beta$ es 0,02 y $T$ es igual a $1, b T$ es igual a 0,98 , un valor muy cercano a la unidad. De esta forma, Quah sugiere que la ecuación (10) puede ser pensada como una prueba de raíz unitaria, y que la concentración de valores de beta en torno al 0,02 sería la aceptación de la misma

En la medición de convergencia de Quah, emerge otro herramental: la evolución de los kernels gaussianos y las matrices de transición de Markov. La importancia de estos instrumentos en lugar de regresiones a la Barro, radica en que las últimas se encuentran también afectadas por la falacia de Galton de reversión a la media. No obstante, Lichtenberg (1994) demuestra que bajo algunos supuestos la tasa de convergencia 
es independiente del grado de reversión a la media. Aunque autor sostiene que, bajo otros supuestos adicionales, la reversión a la media, es en realidad, una condición necesaria pero no suficiente para la convergencia. Sobre una muestra del producto bruto per cápita para 22 países de la OCDE entre 1960 y 1985, el autor provee evidencia empírica en la cual la hipótesis nula de no reversión a la media es rechazada pero la hipótesis nula de no convergencia no es rechazada.

Si el logaritmo del PBI per cápita, representado por Y, es una variable aleatoria que se distribuye a partir de una función de densidad $\mathrm{f}(\mathrm{y})$, la estimación kernel de la función de densidad en un punto y viene representado por:

$$
\hat{f}(y, h)=\frac{1}{n h} \sum_{i=1}^{n} K\left(\frac{y-Y_{i}}{h}\right)
$$

Donde $n$ representa la cantidad de observaciones, $h$ el ancho de banda e y la referencia utilizada para establecer a cuánto dista cada observación $Y_{i}$ de $y$. El kernel gaussiano, $K$, viene determinado por:

$$
K(u)=\frac{1}{\sqrt{2 \pi}} e^{-0.5 u^{2}}
$$

La estimación de la función de densidad dependerá fundamentalmente del número de observaciones, $n$, y del ancho de banda o parámetro de suavizamiento, $h$. Como $n$ queda perfectamente determinado por el tamaño de la muestra, lo que resulta trascendental es la adecuada elección del valor de $h$ para obtener una correcta estimación de la ecuación (12). Un valor demasiado alto para $h$ puede derivar en una densidad estimada unimodal, en tanto que un valor reducido para $h$ podría conducir a una función de densidad estimada con múltiples modas.

En ese sentido, la adecuada elección del valor del parámetro de suavizamiento es uno de los aspectos de mayor relevancia en la metodología propuesta por Quah $(1995,1996)$, para no obtener resultados espurios. La importancia del estudio del kernel es observar si el mismo evoluciona hacia una distribución unimodal, en cuyo caso podría asumirse convergencia, o por el contrario, hacia una distribución multimodal. También Bianchi (1997) ha estudiado el fenómeno de la convergencia a partir de pruebas no paramétricas para testear la multimodalidad.

La contrapartida analítica de los kernels gaussianos son las cadenas de transición de Markov. Las mismas, permiten establecer estados de partida y estados de llegada para los países involucrados en el análisis. Asimismo, el vector ergódico asociado a la matriz de transición, permite conocer, independientemente del estado inicial que ocupaba cada país en el período inicial y suponiendo que la matriz de transición se mantiene vigente para todo el período de análisis, cómo es la distribución de países por grupos de ingresos. Esto es, si la distribución ergódica es normal, la principal implicancia es que podría aceptarse la hipótesis de convergencia incondicional como suceso dominante durante el período de estudio, más allá de la existencia de sub períodos divergentes. Rabanal (2012), arriba a la conclusión de convergencia utilizando este procedimiento para un grupo de 101 países en el período 1950-2009. En dicho estudio, el autor utiliza una matriz de transición de un paso con cuatro estados y otra con cinco estados.

Finalmente, resulta factible cuantificar la movilidad implícita en la matriz de transición. Para lograrlo, es preciso utilizar dos índices propuestos por Quah:

$$
\mu_{1}(P)=(K-1)^{-1}\left[K-\sum_{i=1}^{k} \lambda_{i}\right]
$$




$$
\mu_{2}(P)=1-\lambda_{2}
$$

Siendo $P$ la matriz de transición, $K$ la cantidad de valor propios asociados a $P$ y $\lambda_{2}$ el segundo valor propio más alto. $\mu_{1}(P)$ refiere a la velocidad con la que el proceso transita hacia su estado estacionario -que en este caso es el estado ergódico proveniente del vector ergódico- y $\mu_{2}(P)$ mide la velocidad con la que el desequilibrio de corto plazo es corregido.

El uso de matrices de transición ha recibido algunas críticas por parte de algunos autores, en virtud de que las mismas conducen de manera necesaria a la conclusión de convergencia absoluta (Chumacero, 2001).

\subsection{Convergencia estocástica}

El análisis de las series de tiempo ha proporcionado otras herramientas diferentes a las del análisis transversal, que configuran un marco alternativo para analizar el fenómeno de la convergencia, definiéndola bajo parámetros diferentes. Son habituales bajo este enfoque dos definiciones de convergencia: una más débil y otra más fuerte. De acuerdo con la primera, la convergencia estocástica tiene lugar si el logaritmo de los productos relativos es estacionario en tendencia. Esta propuesta formulada por Carlino y Mills (1993) no estuvo exenta de críticas, ya que la tendencia temporal permite diferencias permanentes en el producto per cápita. Por esta razón, dos años más tarde, Bernard y Durlauf (1995) propusieron una definición más fuerte, en la que la convergencia estocástica ocurre si el logaritmo del producto relativo es estacionario en nivel con media igual a cero. En términos analíticos, esto última definición implica que:

$$
\lim _{t \rightarrow \infty} \mathrm{E}\left(\mathrm{y}_{j, t}-\mathrm{y}_{i, t} \mid I_{0}\right)=0
$$

siendo $i$ y $j$ dos economías diferentes, $t$ un período de tiempo fijo, $I_{0}$ el conjunto de información disponible en el período 0. En consecuencia, la convergencia estocástica ocurrirá si la diferencia entre el logaritmo per cápita de la medida de referencia adoptada y el logaritmo per cápita para un agrupamiento de países sigue un proceso estacionario. En este sentido, habitualmente adquiere relevancia la prueba de DickeyFuller (ADF), dada por la siguiente especificación:

$$
\Delta y_{i t}=\alpha_{i}+\gamma_{i} t+\rho_{i} y_{i, t-1}+\sum_{j=1}^{K} \delta_{i j} \Delta y_{i, t-j}+\varepsilon_{i t}
$$

La expresión (17) podría ser utilizada para testear la hipótesis de convergencia entre diferentes regiones. Li y Papell (1999) han encontrado evidencia de convergencia estocástica para 16 países de la OCDE empleando el enfoque descripto con la incorporación de quiebres estructurales. No obstante, la controversia bajo el enfoque de convergencia estocástica no es menor a la de los estudios de sección cruzada. Es que también han surgido estudios proponiendo divergencia entre los países de la OCDE y otras economías europeas, como el de Flessig y Strauss (2001). En este sentido, autores como Arbia (2004) atribuyen esos resultados al ya conocido problema de la baja potencia en las pruebas de raíz unitaria, siendo este uno de los mayores desafíos que ha atravesado el enfoque de la convergencia estocástica.

\subsection{El papel de la Econometría Espacial en los procesos de convergencia}

La teoría de los centros de desarrollo ha constituido uno de los principales fundamentos para el análisis espacial de los fenómenos económicos. En este sentido, los aportes teóricos más significativos han provenido desde la Teoría del Desarrollo Económico y de la denominada Teoría de la Organización Espacial (Posada, 1978).

Dentro de la Teoría del Desarrollo han existido básicamente dos posturas opuestas en lo relativo al crecimiento. Por un lado, algunos autores como Cassel (1927), Nurske (1953), Rosenstein-Rodan (1943) 
y Lewis (1965) han sido los pioneros en difundir razones a favor de un crecimiento balanceado en las diferentes escalas geográficas que pueda pensarse un territorio. Por otra parte, y en contraposición a éstos, Perreux (1955), Myrdal (1957) y Hirschman (1958) han sugerido la noción de crecimiento económico desequilibrado.

La principal línea de argumentación por parte de los teóricos del crecimiento equilibrado o balanceado tiene un fuerte correlato con la idea de convergencia incondicional, ya que, si el crecimiento es similar en todas las áreas de estudio, y no hay razones para partir de diferentes niveles de renta -o las que pudieran existir desaparecen con el tiempo-, con lo que la convergencia está garantizada en todo momento. No obstante, la noción de crecimiento desequilibrado encuentra congruencia con otras hipótesis alternativas a la de convergencia absoluta, como son la convergencia condicional, la divergencia y los clubes de convergencia. En este sentido, los argumentos de causación circular acumulativa como los de Myrdal (1957) ya reflejaban y sugerían la conocida frase de Quah (1995) “...the poor getting poorer, and the rich richer...".

Otro importante fundamento para incluir el análisis espacial tiene que ver con la difusión espacial de las innovaciones y el conocimiento. Esto es, incorporar al análisis los posibles patrones espaciales para la difusión de invenciones productivas que permitan expandir la frontera de posibilidades de producción. Precisamente Abramovitz (1986), que se mostró a favor de la convergencia, resaltó la necesidad de que los países menos desarrollados cuenten con capacidad social de absorber tecnologías, para poder imitarlas a un costo bajo.

También la Teoría de los Lugares Centrales ha constituido un esfuerzo por comprender las razones por las que las actividades se desarrollan en un lugar en particular y no en otro. Aunque el tema de la localización y la influencia ejercida en las actividades económicas ya había sido abordado por Von Thünen en el siglo XIX, la Teoría de los Lugares Centrales es frecuentemente estudiada de manera independiente (Posada, 1978). Los principales precursores fueron Christaller (1933) y Lösch (1940). Christaller (1933) comienza su análisis estableciendo jerarquías para los diferentes lugares centrales. Para ello, les asigna una importancia relativa ordinal, según las funciones económicas que mantengan con su región complementaria - la que podría ser entendida como zona de influencia-. El resultado final de su modelo es un ordenamiento hexagonal del espacio, y es producto de dos principios: maximización de la distribución de bienes y servicios sujeto a la minimización del número de lugares centrales y optimización en la distribución de lugares centrales, que sólo puede tener lugar cuando los mismos se encuentran sobre rutas diagramadas con el criterio de menor costo y que comunican centros de mayor orden. El análisis de Lösch (1940), en cambio, deriva en un equilibrio de ubicaciones geográficas, puesto que a diferencia de Christaller (1933) comienza sus análisis por centros de menor orden donde no existen necesariamente una progresión jerárquica de lugares centrales (Posada, 1978).

Actualmente, la relación entre el espacio y el crecimiento es abordada generalmente desde dos marcos teóricos: la Nueva Geografía Económica (Krugman, 1991) y la Nueva Teoría del Crecimiento. Desde el primer enfoque se señala a la concentración geográfica como una fuente de ventajas competitivas. Esto da lugar a la formación de aglomerados espaciales, más allá de que en un instante inicial de estudio existan o no. Es decir, aun partiendo de una variable distribuida aleatoriamente en el espacio, siempre existirá un mecanismo capaz de generar aglomeraciones frente a un shock. Por lo tanto, la distribución espacial final de las actividades económicas no resultará azarosa.

La segunda perspectiva ha centrado la atención en la dimensión espacial del crecimiento económico. Los métodos de la econometría espacial "han permitido analizar las implicancias de estas nuevas aproximaciones teóricas” (Braüninger y Niebuhr, 2005: p. 1). En particular, estudios como los de Flingeton (2003) y Funke y Niebuhr (2005) examinan el impacto de dependencia espacial sobre las disparidades en 
el crecimiento. Asimismo, el desarrollo de programas de computación capaces de procesar información geoestadística, le ha dado un gran impulso a esta corriente.

Este avance en las formulaciones teóricas del crecimiento económico, junto a las técnicas de la econometría espacial, han hecho más evidente la necesidad de incorporar las relaciones de dependencia espacial en el análisis, para evitar estimaciones sesgadas por omisión de variables relevantes (Rey y Montouri, 1999; Corrado y Fingleton, 2010). En consecuencia, es el modelo $\beta$-convergencia incondicional con reconocimiento de efectos espaciales el que resultaría más adecuado para representar al fenómeno de estudio. A pesar de esto, las aplicaciones empíricas que consideran efectos espaciales a nivel de países son escasas. En este sentido, las investigaciones tienden a realizar el análisis en el marco regional. La relevancia de las mismas, están relacionadas con el estudio del efecto aglomeración (Ciccone, 2002; Braüninger y Niebuhr, 2005) y las implicancias de los procesos de integración (Kosfeld et. al., 2002). Para el caso de países, Rabanal (2012), estudia un grupo de 101 países, encuentra convergencia a la Barro para el grupo de países de mayor renta, y observa que el reconocimiento de efectos espaciales con dependencia espacial residual, que, al ser adicionados al análisis clásico de convergencia, redundan en una mejora de la bondad del ajuste, coeficientes beta más significativos y mayor velocidad de convergencia.

La presencia de un componente espacial puede manifestarse básicamente de dos maneras. La primera de ellas es a partir de la inestabilidad estructural. Es este caso, una correcta especificación debería considerar parámetros cuyos valores se vean afectados según la localización. Algunos autores como Funke y Niebuhr (2005) consideran a la heterogeneidad espacial como indicativa de efectos umbrales (threshold externalities) que podrían ser capaces de generar equilibrios múltiples en la dinámica de crecimiento.

En términos analíticos, el análisis espacial puede sintetizarse mediante una matriz de contactos W, que recoge los pesos específicos. Su inclusión se da básicamente de dos formas diferentes en la ecuación de convergencia, según la estructura de dependencia espacial existente. Para el caso de la dependencia espacial sustantiva (también conocido como "modelo lag") sería de la siguiente forma:

$$
\frac{1}{T}\left[\log \left(y_{i, T}\right)-\log \left(y_{i, 0}\right)\right]=\alpha+\rho W\left\{\frac{1}{T}\left[\log \left(y_{i, T}\right)-\log \left(y_{i, 0}\right)\right]\right\}+\beta \log \left(y_{i, 0}\right)+\varepsilon_{i t}
$$

donde $\rho$ el coeficiente de autocorrelación espacial.

Por otra parte, la dependencia espacial subyacente puede ser residual, en cuyo caso la correcta especificación vendría dada por el siguiente modelo de "error espacial":

$$
\begin{gathered}
\frac{1}{T}\left[\log \left(y_{i, T}\right)-\log \left(y_{i, 0}\right)\right]=\alpha+\beta \log \left(y_{i, 0}\right)+\varepsilon_{i t} \\
\varepsilon_{i t}=\lambda W \varepsilon_{i t}+\mu_{i t}
\end{gathered}
$$

En este caso la autocorrelación espacial es recogida por $\lambda$ a través del término de perturbación del modelo. Se asume también que $\mu \mathrm{i} \approx i . i . d$. $(0, \sigma 2 \varepsilon)$.

Esta expresión permite modelizar y cuantificar los denominados efectos de difusión espacial del crecimiento. Para ello resulta útil manipular la expresión (19), reformulándola tal como propone Toral Arto (2001):

$$
\log \left(y_{i, T}\right)-\log \left(y_{i, 0}\right)=\text { cons }+\beta \log \left(y_{i, 0}\right)+\rho W\left[\log \left(y_{i, T}\right)-\log \left(y_{i, 0}\right)\right]+\gamma W \log \left(y_{i, 0}\right)+\mu_{i t}
$$

con $\rho=\lambda, \gamma=-\lambda \beta$ y $\mu_{i t} \sim N(0, \sigma 2)$. De esta forma, el modelo expuesto en (20) permite desglosar el efecto espacial en dos. Por un lado, $\rho$ recoge la influencia de las tasas de crecimiento de las regiones contigüas 
sobre la de la región $i$ a partir de una matriz $W$ determinada. Por otra parte, $\gamma$ incorpora el efecto de los valores iniciales de las regiones contigüas sobre la tasa de crecimiento de la región $i$.

Existen diferentes contrastes diseñados para detectar la posible existencia de dependencia espacial, y en tal caso su tipología. Para el testeo de dependencia espacial sustantiva son habituales los contrastes basados en los multiplicadores de Lagrange, el LM-LAG propuesto por Anselin (1988) y el test LM-LE de Bera y Yoon (1992). El LM-LE es robusto frente a la existencia de un término de perturbación correlacionado espacialmente. Para la dependencia espacial residual es frecuente la utilización de los contrastes LM-ERR (Burridge, 1980) y su versión robusta LM-EL (Bera y Yoon, 1992), también basados en los multiplicadores de Lagrange. La ventaja del test LM-EL es que resulta robusto ante posibles especificaciones erróneas locales como la presencia de una variable endógena retardada espacialmente (Moreno y Vayá, 2000: p. 82).

\section{CONCLUSIONES}

El interés en analizar los procesos de convergencia entre diferentes regiones radica en la evidencia que sus resultados pueden proporcionar para el debate de las implicancias vaticinadas por los modelos de crecimiento endógeno y los de crecimiento exógeno. No obstante, la multiplicidad de pruebas desarrolladas para testear el fenómeno ha suministrado gran cantidad de evidencia contradictoria entre sí, siendo posible encontrar evidencia a favor de convergencia absoluta, de la convergencia condicional e incluso de la divergencia. Los análisis más usuales van desde análisis de series de tiempo hasta modelos de datos de panel, pasando por modelos de sección cruzada.

En este trabajo, se realiza una diferenciación de las hipótesis alternativas que habitualmente se realizan sobre la convergencia, a partir de una revisión de la evidencia empírica que soporta a cada una de ellas. La detección de fortalezas y debilidades de cada uno del procedimiento empleados para medir la convergencia económica resulta crucial para obtener resultados fiables. Las principales debilidades de las regresiones a la Barro son su carácter estático, lo que imposibilita el estudio de la dinámica de convergencia, y la reversión a la media. Por otra parte, la metodología de Quah queda sujeta a la determinación de un ancho de banda óptimo, y a la elección de un número de estados para la construcción de las matrices de transición dinámica, lo que encierra cierto grado de subjetividad. Finalmente, los enfoques de convergencia estocástica se enfrentan al ya conocido problema de la baja potencia en las pruebas de raíz unitaria Si bien todos los métodos se encuentran vigentes en la actualidad, existe, desde hace aproximadamente diez años, un fuerte interés en el estudio de los efectos derivados de la dependencia espacial. Este auge puede explicarse fundamentalmente en el desarrollo de software geoestadístico especializado, lo que ha concedido un gran impulso al estudio de los efectos umbrales y las externalidades en los procesos de convergencia. 


\section{REFERENCIAS BIBLIOGRÁFICAS}

Abramovitz, M. (1986): "Catching Up, Forging Ahead, and Falling Behind", The Journal of Economic History, Vol. 46, pp.385-406.

Álvarez, Lucas Santos, S. y Delgado, Ma. de Jesús (2009): “Globalización y convergencia

económica: Un análisis empírico para los países de América Latina" en Paradigma Económico, Vol. 1, pp. 40-59.

Anselin, L. (1988): "Lagrange Multiplier Test Diagnostic for Spatial Dependence and Spatial Heterogeneity", Geographical Analysis, Vol. 20-1, pp. 1-17.

Arbia, G. y Deglistudi A. (2004): "Alternative Approaches to Regional Convergence Exploiting both Spatial and Temporal Information", Estudios de Economía Aplicada, Vol. 22-3, pp. 431-450.

Barro, R. J. (1991): "Economic Growth in a Cross Section of Countries", The Quarterly Journal of Economics, Vol. 106-2, pp. 407-443.

Barro, R. J. y Sala-I-Martin X. (1991): "Convergence Across States and Regions". Brooking Papers on Economic Activity, pp. 107-182.

Barro, R. J. y Sala-I-Martin X. (1992): “Convergence” en Journal of Political Economy, Vol. 100-2, pp. 223-251.

Baumol, W. (1986): "Productivity Growth, Convergence, and Welfare: What the Long-Run Data Show", The American Economic Review, Vol. 76-5, pp. 1072-1085.

Ben-David, D. (1997): "Convergence Clubs and Diverging Economies". CEPR, Working Paper N 922.

Bera, A. K. y Yoon, M. J. (1992): "Simple Diagnostic test for spatial dependence", University of Illinois, Department of Economics (mimeo). Champaing, IL: Department of Economics, University of Illinois.

Bernard, A. y. Durlauf, S. N. (1995): "Convergence in International Output", en Journal of Applied Econometrics, Vol.10, pp. 97-108.

Bernard, A.B. y Durlauf, S.N. (1996): "Interpreting tests of the convergence hypothesis", Journal of Econometrics, Vol. 71, pp. 161-173.

Bianchi, M. (1997): "Testing for Convergence: Evidence from Non-Parametric Multimodality Tests", Journal of Applied Econometrics, Vol. 12-4, pp. 393-409.

Boyle, G.E. y McCarthy, T.G. (1997): "A Simple Measure of Beta Convergence", en Oxford

Bulletin of Economics and Statistics, Vol. 59(2), pp. 257-264.

Boyle, G.E. y McCarthy, T.G. (1999): "Simple Measures of Convergence in Per Capita G D P :

A Note on Some Further International Evidence", en Applied Economics Letters, Vol. 6(6), pp.343-347.

Braüninger, M. y Niebuhr, A. (2005): "Agglomeration, Spatial Interaction and convergence in the EU", HWWA Discussion Paper, 322. Hamburg.

Burridge, P. (1980): “On the Cliff-Ord Test for Spatial Autocorrelation”, Journal of the Royal Statistical Society B, Vol. 42, pp. 107-108.

Carlino, G. A., y Mills, L. O. (1993). "Are U.S. regional incomes converging? A time series analysis" en Journal of Monetary Economics, Vol. (32), pp. 335-346.

Carvalho, V. M y Harvey, A. C. (2005): "Convergence in the Trends and Cycles of euro-zone Income", Journal of Applied Econometrics, Vol. 20, pp. 275-289.

Cassel, G. (1927): Theoretische Sozial Ekonomic. Leipzig.

Chenery, H. y Syrquin, M. (1986): "Typical Patterns of Transformation" in Hollis Chenery, Sherman Robinson y Moshe Syrquin, eds. Industrialization and Growth: A Comparative Study, Oxford: 
Oxford University Press.

Christaller, W. (1933): "Die Zentralen Orten in Süddeutschland: Eine ökonomisch-geographische Unterschung über die Gesetzmässigkeit der Verbreitung und Entwicklung der Siedlungen mit städtischen Functionen”. Jena. Traducción al ingles: Baskin, Carlisle. Publicado como “Central Places in Southern Germany". Englewood Cliffs, New Jersey, 1966.

Chumacero, R. A. (2001): "Absolute Convergence, Period", Quinta Conferencia Annual del Banco Central de Chile. Department of Economics of the University of Chile and Research Department of the Central Bank of Chile. Pp. 1-33.

Ciccone, A. (2002): “Agglomeration-Effects in Europe”, European Economic Review, Vol. 46, pp. 213247.

Corrado, L. y Fingleton, B. (2011): "Where is the Economics in the Spatial Econometrics", Discussion Papers in Economics, $\mathrm{N}^{\circ}$ 11-01, pp. 1-34.

DeLong, J.B. (1988): "Productivity Growth, Convergence, and Welfare: Comment," American Economic Review, Vol. 78-5, pp. 1138-1154.

Dollar, D. y Wolff E. (1994): "Capital Intensity and TFP Convergence in Manufacturing, 1963-1985", en William J. Baumol, Richard R. Nelson, and Edward N. Wolff, eds., Convergence of Productivity: Cross National Studies and Historical Evidence. New York, Oxford University Press.

Dollar, D. (2001): “Globalization, Inequality and Poverty since 1980”, working paper, World Bank, pp. $1-39$.

Dreher, A. (2006): "The influence of globalization on taxes and social policy: An empirical análisis for OECD countries", en European Journal of Political Economy, Vol. 22, pp. 179-201.

Esteban, J.M. y Ray D. (1994), “On the measurement of polarization”, en Econometrica, N 4, pp. 819-851.

Funke, M. y Niebuhr, A. (2005): "Regional Geographic R\&D Spillovers and Economic GrowthEvidence from West Germany", Regional Studies, Vol. 39, pp. 143-154.

Galor, O. (1996)-. "Convergence? Inferences from Theoretical Models", The Economic Journal, Vol. 106, pp. 1056-1069.

Hirschman, A. O. (1958): The Strategy of Economic Development, Yale University Press, New Haven. Islam, N. (1995): “Growth Empirics: A Panel Data Approach”, Quarterly Journal of Economics, Vol. 110, pp. 1127-1170.

Islam, N. (2003): "What have We Learnt from the Convergence Debate?", Journal of Economic Surveys, 17(3), 309-362.

Kosfeld, R., Ecke, H. y Greger, C. (2002): "Regional convergence in unified Germany, A spatial econometric perspective". Nomos-Press Series Edition 19.

Kristensen, T. (1982): "Development in Rich and Poor Countries”, New York: Praeger.

Krugman, P. (1991): "Increasing returns and economic geography”, Journal of Political Economy, Vol. 99, pp. 483-499.

Lee, K. M., Pesaran, H. y Smith R (1997): "Growth and Convergence: A Multicountry Empirical Analysis of the Solow Growth Model", Journal of Applied Econometrics, Vol. 12, pp. 357-392.

Lichtenberg, F. R. (1994): "Testing the Convergence Hypothesis" en The Review of Economics and Statistics, Vol. 76 (3), pp. 576-579.

Lindert, P. H. y Williamson, J. G. (2003): “Does Globalization Make the World More Unequal?”, NBER, pp. 227-275.

Li, Q. y Papell, D. (1998): “Convergence of International Output. Time Series evidence for 16 OCDE Countries" en International Review of Economics and Finance, Vol. 8, pp. 267-280. 
Li, Q. (1999): “Convergence Clubs: Some Further Evidence” en Review of International Economics, Vol. 7 (1), pp. 59-67.

Loayza, N. y Fajnzylber, P. (2005): Economic Growth In Latin America And The Caribbean: Stylized Facts, Explanations, And Forecasts, World Bank Publications.

Lucas, R. (1988): "On the Mechanics of Economic Development", Journal of Monetary Economics, Vol. 22-1, pp. 3-42.

Mankiw, G., Romer, D y Weil, N. (1992): "A Contribution to the Empirics of Economic Growth", Quarterly Journal of Economics, Vol. 107, 407-413.

Moreno, R. y Vayá, E. (2000): Técnicas econométricas para el tratamiento de datos espaciales: La econometría espacial. Edicions Universitat de Barcelona.

Myrdal, G. (1957): Rich Land and Poor, Harper and Row, New York.

Nurske, R. (1953): Problems of Capital Formation in Developing Countries, Oxford University Press, Oxford.

Perroux, F. (1955): "Note sur la notion de Pole de Croissance”, Economie Apliquée, № 1, pp. 307-320.

Posada, L. J. (1978): "Fundamentos económicos-espaciales de la teoría de Centros de Desarrollo", Agricultura y Sociedad, No 6, Ministerio de Agricultura y Comercio de España. Pp. 137-180.

Quah, D. (1993): “Galton's Fallacy and Tests of the Convergence Hypothesis”, en The Scandinavian Journal of Economics, Vol. 95-4, pp. 427-443.

Quah, D. T. (1995): "Empirics for Economic Growth and Convergence", Centre for Economic Performance, Discussion Paper $N^{\circ} 253$, pp. 1-23.

Quah, D. (1996): “Twin Peaks: Growth and Convergence in Models of Distribution Dynamics" en The Economic Journal, Vol. 106-43, pp. 1045-1055.

Rabanal, C. (2012): “Análisis de la convergencia económica internacional en el período 1960-2009” en Revista de Economía Mundial, Vol. 31, pp. 167-197.

Rebelo, S. (1991):" Long Run Policy Analysis and Long Run Growth", Journal of Political Economy, pp. 500-521.

Rey, S. y Montouri, B. (1999): "US Regional Income Convergence a Spatial Econometrics Perspective". Regional Studies, pp. 145-156.

Rodrick, D. (2011a): "The Future of the Economic Convergence", Jackson Hole Symposium of the Federal Reserve Bank of Kansas City, pp. 1-49.

Rodrick, D. (2011b): “Unconditional Convergence”, NBER Working Paper 17546, pp. 1-39.

Romer, P. (1986): “Increasing Returns and Long-Run Growth”, Journal of Political Economy, Vol. 945, pp. 1002-1037.

Rosenstein-Rodan, P. N. (1943): "Problems of Industrialization of Eastern and Southern Europe", Economic Journal, Vol. 53, pp. 202-211.

Rostow, W. W. (1980): "Why the Poor Get Richer and the Rich Slow Down”, Austin, TX: University of Texas Press.

Sachs, J. D. y Warner, A. M (1995): “Economic Convergence and Economic Policies” en NBER Working Papers Series, N 5039, pp. 1-47.

Sala-i-Martin (1996): "Regional Cohesion: Evidence and Theories of Regional Growth and Convergence", European Economic Review, Vol. 40, pp. 1325-1352.

Silverman B. W. (1986). Density Estimation for Statistics and Data Analysis. London, UK: Chapman \& Hall.

Solow, R. T. (1956): “A Contribution to the Theory of Economic Growth", Quarterly Journal of 
Economics, Vol. 70, pp. 65-94.

Spence, M. (2011): The Next Convergence: The Future of Economic Growth in a Multispeed World. Nueva York: Farrar, Straus and Giroux.

Swan, T. (1956): “Economic Growth and Capital Accumulation”. Economic Record, XXXII, pp. 334361.

Toral Arto, M. A. (2001): El factor espacial en la convergencia de las regiones de la Unión europea: 1980-1996. Tesis doctoral. ISBN: 84-689-0568-2. Madrid. España.

Villaverde, J. y Maza, A. (2011): “Globalization, Growth and Convergence”, en The World Economy, Vol. 34(6), pp. 952-971.

Villaverde, J. (2004): “Indicators of Real Income Convergence. A Primer” en UNU-CRIS e-Working Papers, pp. 1-25. 